\section{Absence of age-related optic disk changes in young children with optic nerve hypoplasia}

The Vision Center, Children's Hospital Los Angeles, Los Angeles, CA 90027, USA

Correspondence: M Borchert, The Vision Center, Children's Hospital Los Angeles, 4650 Sunset Blvd., Los Angeles, CA 90027, USA

Tel: +1 323361 4510;

Fax: +1 3233613512 .

E-mail: mborchert@

chla.usc.edu

Received: 10 September 2013

Accepted in revised form:

12 December 2013

Published online:

21 February 2014
Abstract

Purpose To determine whether the ratio of optic disk diameter to disk-to-macula distance (DD/DM) in children with optic nerve hypoplasia $(\mathrm{ONH})$ changes over time. Patients and methods Fifteen subjects (29 eyes) enrolled in a prospective registry study on ONH had fundus photography performed under the age of 24 months and again at 60 months. Using the ratio of the DD/DM method, the relative size of the optic disk was assessed twice for each photo by one masked expert examiner to determine whether any change in relative disk size occurred over time.

Results There was no change in relative optic disk size between initial and final fundus photographs. The average ( \pm SD) age at the time of initial and final fundus photography was, respectively, 11.6 \pm 5.2 months and $60.6 \pm 1.3$ months. Strong concordance was noted between the average DD/DM of the initial and the final photographs ( $\rho=0.939 ; 95 \%$ CI: $0.893,0.981)$. There was negligible difference between the individual time point measurements $(-0.011 \pm 0.03)$ (95\% LOA: $-0.07,0.04)$. Conclusion There were no clinically significant changes in relative optic disk size over time in children with $\mathrm{ONH}$; thus, $\mathrm{DD} /$ DM measurements need not be adjusted by age beyond 1 year in children with this disorder.

Eye (2014) 28, 562-566; doi:10.1038/eye.2014.12; published online 21 February 2014

Keywords: optic nerve hypoplasia; DD/DM; optic disk measurement
J Sutedja, P Garcia-Filion, C Fink and M Borchert

\section{Introduction}

In the United States and Europe, optic nerve hypoplasia $(\mathrm{ONH})$ has emerged as the leading ocular cause of childhood blindness with a prevalence estimate of 1.1 per 10000 children. ${ }^{1}$ The defining feature of $\mathrm{ONH}$ is underdevelopment of the optic nerve in one or both eyes.

It is often associated with endocrine dysfunction, delays in neurocognitive development, seizures, autism, dysregulation of the hypothalamus, and neurological abnormalities. ${ }^{2-4}$

Optic nerve size is used to approximate severity of disease and vision potential in children with $\mathrm{ONH} .{ }^{5}$ This can be done subjectively through indirect ophthalmoscopy or objectively by measuring the relative optic disk size on fundus photography. A study by De Silva et al, ${ }^{6}$ reporting $51 \%$ growth in the optic disk relative to other fundus landmarks between birth and adulthood, raises the question of whether such optic disk measurements require adjustment by age.

During the first 3 years of life, there is overall growth of the eye. ${ }^{7}$ However, histologic measurements demonstrate that $95 \%$ of the growth of the optic disk is completed by 1 year of age. ${ }^{8}$ It is therefore unknown whether the size of the optic disk relative to other retinal landmarks changes over time in young children. This is important in the assessment of $\mathrm{ONH}$, for which the relative size of the optic disk may be measured on fundus photography by taking the ratio of the horizontal disk diameter (DD) to the distance from the disk to the macula (DM). ${ }^{9}$ This has been shown to be widely effective in identifying the degree to which an eye is 
affected with $\mathrm{ONH}$, while avoiding the need for correction of magnification effects due to high refractive error. $^{10-12}$

This study aimed to determine whether a change in the relative optic disk size occurs with age, and whether or not variability owing to the measuring technique or photographic quality contributes to relative disk size measurements in young children. Using subjects from a prospective registry study, we measured and compared the DD/DM ratio from fundus photographs between two time points ( $<24$ months of age $v$ s age 60 months) in children with unilateral or bilateral $\mathrm{ONH}$.

\section{Materials and methods}

The Vision Center at the Children's Hospital Los Angeles (CHLA) serves as a referral center for children with $\mathrm{ONH}$ from across the United States. In 1992, a registry study was established to prospectively follow children with this condition in order to identify clinical characteristics at presentation that might predict outcomes at school age and to identify risk factors for having a child with $\mathrm{ONH}$. Methods for enrollment and comprehensive data collection in the study have been previously described., ${ }^{2,3}$

Registry participants undergo baseline fundus photography while sedated with chloral hydrate at an age of $<24$ months. Subjects returning for their final registry visit at age 60 months between April 2009 and December 2011, who were able to cooperate with unsedated fundus photography, were asked to participate in this study by undergoing a second set of fundus photography. Otherwise, previously enrolled registry subjects did not have repeat photographs taken at the age of 60 months. This study was conducted in accordance with approval from the Committee on Clinical Investigations (IRB) at CHLA.

An ophthalmic photographer took the photographs ( $30^{\circ}$ angle) using a Nikon NF-505 Digital Fundus Camera (Nikon Inc., Tokyo, Japan) for the initial photo set and a Canon CF-1 Digital Mydriatic Retinal Camera (Canon Inc., Tokyo, Japan) for the final photo set. A fixation method was not required for the initial set of fundus photographs, as the children were sedated and oriented in a right lateral decubitus position. The orientation of the camera back was similarly rotated $90^{\circ}$ to avoid changes in perspective. For the final (unsedated) photographs, the children were seated in an upright position and a fixation target was used. If the participant was unable to focus on the internal target, an external fixation target for the opposite eye was used. The internal fixation target was removed simultaneously with the flash. Photographs of the left eye were taken first at the initial visit. The eye with better vision was photographed first for the final set.
Two photographs, one per eye, were used for each time point, and relative optic disk sizes were calculated for both images for each participant. Each photograph was measured twice: photographs were measured in a masked manner days to weeks after they were taken, and all were measured again after completion of enrollment and data collection admixed with photos from another study to ensure blinding.

The relative optic disk size (DD/DM) was estimated by a single neuro-ophthalmologist (MB) using OIS WinStation XP software's measurement tool (OIS, Sacramento, CA, USA). Horizontal DD was estimated at the center of the disk, halfway between the superior and inferior poles. Distance from the DM was measured from the temporal terminus of the DD line to the fovea (Figure 1). When a foveal umbo was not clear, the center of the macula was estimated at the center of the macular pigment. In the event that one photograph did not provide an adequate view of both the macula and the optic disk, adjacent photographs of identical magnitude were montaged using WinStation.

The data were analyzed (Stata SE 11.0, Stata Corporation, College Station, TX, USA) to determine the agreement in the relative optic disc size between time points. Data are summarized using either the mean and $\mathrm{SD}$ or the median with interquartile range (IQR). Agreement was measured using the concordance correlation coefficient $(\rho) .{ }^{13}$ The correlation coefficients are presented with $95 \%$ confidence intervals $(95 \% \mathrm{CI})$. As there was high reproducibility in the repeat measurement of DD/DM ratios within the initial $(\rho=0.976 ; 95 \%$ CI: $0.957,0.994)$ and final $(\rho=0.962 ; 95 \%$ CI: $0.934,0.991)$ set of photographs, a calculated average of the repeated ratios was used for comparison between the time points. Agreement was further examined using the Bland-Altman $95 \%$ limits of agreement $(95 \% \text { LOA })^{14}$ based on the difference and the average of the ratios for the two time points. The spherical equivalent refractive error, DD, and DM measurements for the initial and final time points were examined and compared using the paired $t$-test. Statistical significance was defined as an alpha of 0.05 , with two-sided alternative hypotheses.

The authors certify that all applicable institutional and governmental regulations concerning the ethical use of human volunteers were followed during this research.

\section{Results}

Nineteen subjects were enrolled in the study, and of those, 15 had measurable photos, for a total of 29 eyes. Of the four subjects enrolled without adequate photographs, one did not have an available ophthalmic photographer; two subjects experienced attention issues, inability to fixate on a target, or inability for the photographer to focus 


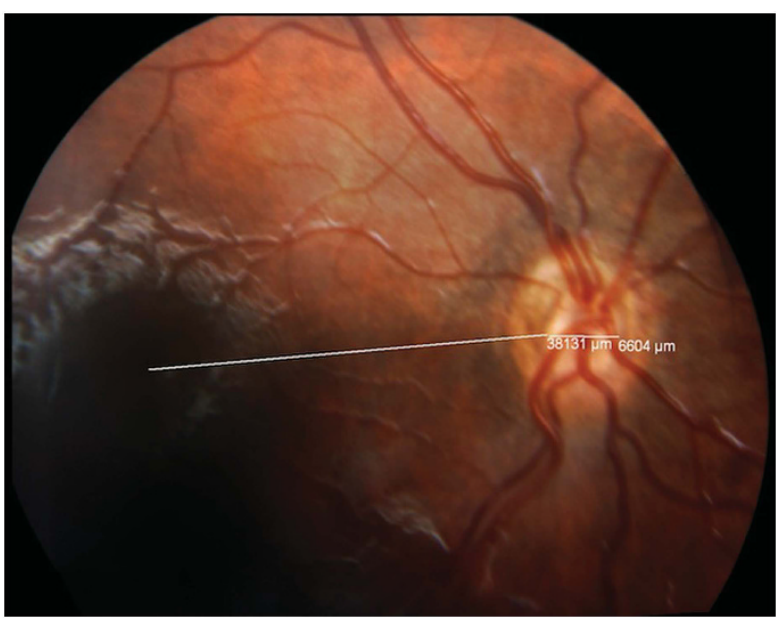

Figure 1 Sample fundus photograph demonstrating measurement of optic DD and distance from DM.

appropriately for a well-framed photo (for example, due to nystagmus); and one subject had initial fundus photography done on film, whereas the final images were taken digitally, resulting in non-comparable measurements.

The average $( \pm S D)$ age at the time of initial and final fundus photography was $11.6 \pm 5.2$ months and $60.6 \pm 1.3$ months, respectively. The median (IQR) spherical equivalents were unchanged between the initial and final visits $(-0.69(-1.5,1)$ vs $-0.50(-1.1,5) ; P=0.745)$. A refractive error $>+4.0$ or $<-4.0$ was noted in both eyes of one subject at the initial visit and four eyes (three subjects) at the final visit.

Strong concordance was noted for the average DD/ DM measurements between the initial and the final sets of photographs ( $\rho=0.939 ; 95 \%$ CI: $0.893,0.981)$. There was negligible difference between time points $(-0.011 \pm 0.03)(95 \%$ LOA: $-0.07,0.04)$. Both the absolute disc size and DM distance increased during the study period, $P=0.022$ and $P=0.040$, respectively.

\section{Discussion}

Analysis of fundus photographs demonstrated two findings: 1) there is high reproducibility in the measurements of DD/DM and 2) there is no clinically significant change in the $\mathrm{DD} / \mathrm{DM}$ ratio between age $<24$ months and age 60 months in children with $\mathrm{ONH}$.

De Silva $e t a l^{6}$ reported an increase in optic nerve size disproportionate to the growth of the eyeball in infants compared with normal adult measurements, with $44 \%$ growth in the optic disk compared with $11 \%$ increase in the distance of the DM. In our cohort, we also observed an increase in the absolute disc size and DM measurements, although this did not impact the DD/DM over time. In addition, premature infants (average gestational age $30.1 \pm 4.7$ weeks) comprised the majority

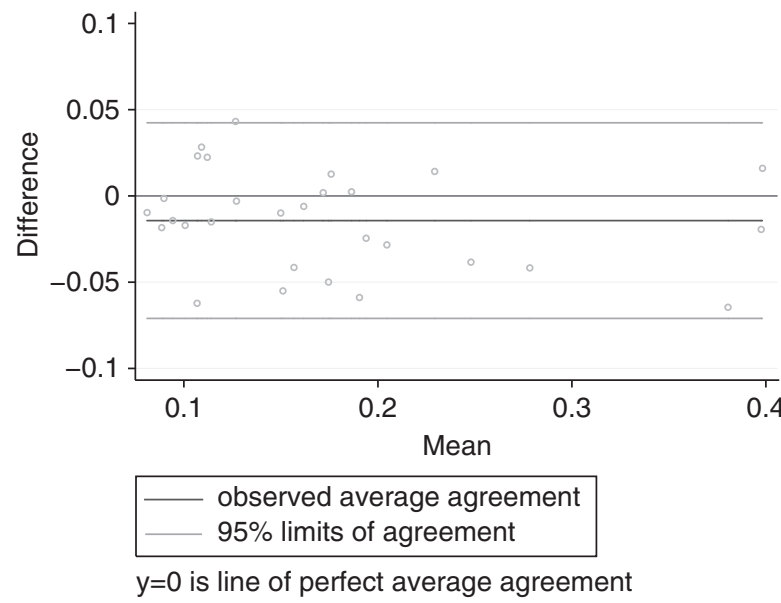

Figure 2 Bland-Altman plot of DD/DM measurements between the initial and final fundus photographs (difference vs average) demonstrating close LOA and no systematic or proportional bias over the measurement range.

of De Silva's patient sample from a neonatal intensive care unit. Prematurity, uncommon in $\mathrm{ONH}(11 \%),{ }^{15}$ was present in just under $7 \%(n=1)$ of our study sample. Contrary to our study methods, the findings by De Silva et $a l^{6}$ were not based on repeat measurements within the same subjects or at different time points; rather, the infants' measurements were compared with normal adult measurements, despite the cohort's prematurity. The only other study measuring optic disk size of infants from fundus photographs also did not assess reproducibility in measurements. ${ }^{16}$

In our cohort of children with $\mathrm{ONH}$, the $\mathrm{DD} / \mathrm{DM}$ ratio did not change between the initial and final photographs (with an average time interval of 48 months). Similarly, the DD/DM was unchanged in the unaffected eyes of three subjects with unilateral $\mathrm{ONH}(\mathrm{DD} / \mathrm{DM}>0.35){ }^{12}$ $\mathrm{DD} / \mathrm{DM}$ measurements were highly reproducible regardless of DD/DM size (Figure 2).

Refractive errors were comparable between time points in our study, with a high refractive error noted in few subjects. There should be little to no magnification effect when the refractive error is within +4.0 and $-4.0 .{ }^{17}$ Excluding subjects outside of this range minimally impacted the concordance; the agreement increased to 0.943 (compared with 0.939).

This study's strengths include sampling of patients from a registry with near-consecutive enrollment of patients with ONH. Diagnostic criteria were uniform, and one masked expert observer diagnosed cases of $\mathrm{ONH}$ and performed measurements. Photographs taken at the later time point were of higher resolution; however, this was not readily discernible to the naked eye, as differences between a low-resolution photograph and a higher-resolution photograph with soft focus were indistinguishable. 
Researchers who use relative optic disc size to categorize severity of disease should recognize that variability in measurements as a result of inconsistent photographs could impact categorization results. We encountered uniformity problems with each set of photos. In the initial set, we speculate that the photo quality was not as good as the final set owing to lowerresolution capabilities of the older camera as well as difficulty with proper framing of photos in children whose eyes were elevated during sedation. The relative blurriness of these photos complicated clear delineation of edges of the optic disk for measurements. In some photos, these landmarks were partially or fully out of frame. Variability in the final set of measurements is likely related to an inability of the participant to fixate while awake, either owing to physical impediments such as nystagmus or attention deficits. In spite of these difficulties, differences in masked measurements performed months apart of the same photos were low.

DD/DM measurement performed by an experienced examiner is a valid technique for measuring optic nerve size in young children that does not require adjustment by age beyond one year. Careful consideration of image quality, framing, and the examiner's ability to identify fundus landmarks should be considered when measuring relative optic disk size.

\section{Summary}

What was known before

- In patients with $\mathrm{ONH}$, optic nerve size is used to approximate severity of disease and vision potential. This is measured by assessing the optic disk subjectively by indirect ophthalmoscopy or objectively with fundus photography.

- There is overall growth of the eye and optic disk between birth and adulthood, suggesting the need to adjust optic disk measurements for age. It is unknown whether the size of the optic disk relative to other retinal landmarks changes over time in young children.

- Furthermore, the reliability of fundus photographic measurements is unknown.

What this study adds

- This study used fundus photography to examine the change in relative optic disk size between two time points ( $<24$ months and age 5 years), and whether the variability is due to measurement technique or photographic quality.

- There was negligible variability within repeat time point measurements and no change between the time point measurements.

- Relative optic disk size measurements do not need to be adjusted for age beyond 1 year when fundus photography is the measurement method.

\section{Conflict of interest}

The authors declare no conflict of interest.

\section{Acknowledgements}

This research was supported in part by the One Small Voice Foundation and Grant No. UL1TR000130, Children's Hospital Los Angeles from the National Center for Advancing Translational Sciences (NCATS) at the National Institute of Health.

\section{References}

1 Patel L, McNally RJQ, Harrison E, Lloyd IC, Clayton PE. Geographical distribution of optic nerve hypoplasia and septo-optic dysplasia in northwest England. J Pediatr 2006; 148(1): 85-88.

2 Garcia-Filion P, Epport K, Nelson M, Azen C, Geffner ME, Fink C et al. Neuroradiographic, endocrinologic, and ophthalmic correlates of adverse developmental outcomes in children with optic nerve hypoplasia: a prospective study. Pediatrics 2008; 121(3): e653-e659.

3 Ahmad T, Garcia-Filion P, Borchert M, Kaufman F, Burkett L, Geffner M. Endocrinological and auxological abnormalities in children with optic nerve hypoplasia: a prospective study. J Pediatr 2006; 148(1): 78-84.

4 Borchert M, Garcia-Filion P. The syndrome of optic nerve hypoplasia. Curr Neurol Neurosci Rep 2008; 8(5): 395-403.

5 Borchert M, McCulloch D, Rother C, Stout AU. Clinical assessment, optic disk measurements, and visual-evoked potential in optic nerve hypoplasia. Am J Ophthalmol 1995; 120(5): 605-612.

6 De Silva DJ, Cocker KD, Lau G, Clay ST, Fielder AR, Moseley MJ. Optic disk size and optic disk-to-fovea distance in preterm and full-term infants. Invest Ophthalmol Vis Sci 2006; 47: 4683-4686.

7 Eustis H, Sprague M, Guthrie E. Postnatal development. In: Wright KW, Spiegel PH (eds). Pediatric Ophthalmology and Strabismus. Springer-Verlag: New York, USA, 2003, pp 39-41.

8 Rimmer S, Keating C, Chou T, Farb MD, Christenson PD, Foos RY et al. Growth of the human optic disk and nerve during gestation, childhood, and early adulthood. Am J Ophthalmol 1993; 16: 748-753.

9 Barr DB, Weir CR, Purdie AT. An appraisal of the disc-macula distance to disc diameter ratio in the assessment of optic disc size. Ophthalmic Physiol Opt 1999; 19(5): 365-375.

10 Alvarez E, Wakakura M, Khan Z, Dutton GN. The disc-macula distance to disc diameter ratio: a new test for confirming optic nerve hypoplasia in young children. J Pediatr Ophthalmol Strabismus 1988; 25(3): 151-154.

11 Zeki SM, Dutton GN. Optic nerve hypoplasia in children. Br J Ophthalmol 1990; 74: 300-304.

12 Borchert MS, McCulloch DL, Rother C, Stout AU. Clinical assessment, optic disk measurements, and visual-evoked potential in optic nerve hypoplasia. Am J Ophthalmol 1995; 120: 605-612.

13 Lin LI. A concordance correlation coefficient to evaluate reproducibility. Biometrics 1989; 45: 255-268. 
14 Bland JM, Altman DG. Statistical methods for assessing agreement between two methods of clinical measurement. Lancet 1986; 1: 307-310.

15 Garcia-Filion P, Fink C, Geffner ME, Borchert M. Optic nerve hypoplasia in North America: a re-appraisal of perinatal risk factors. Acta Ophthalmolol 2010; 88(5): 527-534.
16 Kandasamy Y, Smith R, Wright IMR, Hartley L. Optic disc measurements in full term infants. Br J Ophthalmol 2012; 96: 662-664.

17 Bengtsson B, Krakau CE. Some essential optical features of the Zeiss fundus camera. Acta Ophthalmol (Copenh) 1977; 55: 123-131. 